\title{
Effet d'une surcharge en vitamine $E$, administrée oralement avant abattage, sur la coloration du gras de couverture des carcasses d'agneaux de bergerie
}

\author{
G Bozzolo 1, M Bouillier-Oudot 1, G Phrem 1 \\ Avec la collaboration technique de D Grasset et $\mathrm{H}_{\text {Manse }}{ }^{2}$ \\ ${ }^{1}$ ENSAT (École nationale supérieure agronomique), 145, av de Muret, \\ 31076 Toulouse Cedex; \\ 2 Groupement des éleveurs de brebis du bassin de Roquefort, 12101 Millau, France
}

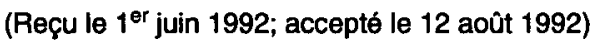

\begin{abstract}
Résumé - L'effet d'une surcharge orale d'acétate de $D L \alpha$-tocophérol administrée à raison de $0,1 \mathrm{~g}$ $\mathrm{kg}^{-1}$ de poids vif, $4 \mathrm{j}$ avant l'abattage, a été contrōlé sur la coloration du tissu gras externe d'agneaux; 240 mâles Lacaunes (120 témoins, 120 traités), sevrés précocement et soumis à un engraissement intensif en bergerie (gain moyen quotidien : $312 \mathrm{~g} \mathrm{j}^{-1}$, poids de carcasse: $19,5 \mathrm{~kg}$ ), avec des caractéristiques d'engraissement externe semblables (épaisseur, recouvrement, fermeté du tissu gras) ont servi à comparer les fréquences des types de coloration observés après $18 \mathrm{~h}$ de ressuage en chambre froide $\left(4^{\circ} \mathrm{C}\right)$. Ceux-ci ont été répertoriés en 7 classes, par référence au nuancier Munsell. La vitaminémie contrólée sur 15 animaux de chaque lot, 3 j après l'administration, montre que la teneur plasmatique est accrue $\left(1,93 \mu \mathrm{g} \mathrm{ml}^{-1}\right.$ vs $\left.0,70 \mu \mathrm{g} \mathrm{ml}-1 ; P<0,001\right)$. Cet apport en vitamine $E$ induit une amélioration de la proportion de carcasses blanc crème $(38 \%$ vs $19 \% ; P<0,001)$ au détriment des carcasses aux nuances bistre-rougeâtre (27\% vs $46 \% ; P<0,002$ ). La proportion de carcasses aux nuances jaunes n'est pas modifiée ( $34 \%$ en moyenne).
\end{abstract}

agneaux / vitamine E / couleur / carcasse

Summary - Effect of vitamin E administered orally before slaughter upon external fat carcass colour of male lambs raised indoors. The effect on the external fatty tissue colour from lambs of oral administration of oL $\alpha$-tocopherol acetate $\left(0.1 \mathrm{~g} \mathrm{~kg}^{-1}\right.$ live weight), 4 days before slaughtering was investigated. Lacaune males $\mathrm{N}=240: 120$ reference group; 120 treated group), weaned early and intensively fed indoors (average daily gain: $312 \mathrm{~g} \mathrm{~d}^{-1}$; carcass weight: $19.5 \mathrm{~kg}$ ) and with similar fat cover characteristics (subcutaneous fatty tissue depth, development and firmness) were compared by colour score (7 grades scaled with Munsell's toner). Scoring was carried out-18-h on chilled carcasses $\left(4^{\circ} \mathrm{C}\right)$. Plasma vitamin $E$ concentration tested in 15 lambs from each group, $3 d$ af-

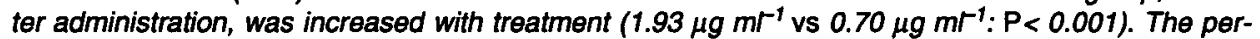
centage of creamy white carcasses was therefore higher (38\% vs $19 \% ; P<0.001)$ and the percentage of reddish-bistre colour lower (27\% vs $46 \%$; $\mathrm{P}<0.002$ ). The percentage of yellow tones was the same in both groups (34\% on average). 


\section{INTRODUCTION}

L'inventaire des couleurs observées sur le tissu gras externe de carcasses d'agneaux, ainsi que les causes probables de leur genèse ont été largement analysés (Rozier et al, 1979; Kirton et al, 1975; Prache et al, 1990). Chez l'agneau mâle de Roquefort, sevré précocement (4 à 5 sem) et nourri intensivement en bergerie avec un aliment concentré, la nuance de couleur bistre-clair (réf: 7.5YR8/6 du nuancier Munsell) apparaît sur plus de $25 \%$ des carcasses (Bozzolo et al, 1991). En raison de l'apparition de ce type de coloration, en partie au cours du ressuage, sur des carcasses caractérisées par des gras mous et huileux, l'hypothèse d'une oxydation des graisses insaturées a été souvent avancée. En effet, la formation de peroxydes colorés et de lipopigments brun-jaune associés (lipofuscine, pigments céroïdes) pourrait rendre compte de cette évolution (Leboulanger, 1977; Lehninger, 1979; Palmer et al, 1986).

Sous ce rapport, la protection conférée par la vitamine $E$ contre l'oxydation des graisses a été surtout étudiée chez les volailles, en relation avec le rancissement des viandes durant la conservation (Rethwill et al, 1981; Bartov et al, 1983). Cependant, Buckley et al (1989) chez le Porc, Faustman et al (1989) chez le Bouvillon, ont également enregistré les effets positifs d'une supplémentation en vitamine $E$ sur la limitation post-mortem de l'oxydation des lipides. Chez le mouton, l'effet de cet antioxydant a été peu étudié. Murphy et l'Estrange (1977), sur un faible nombre d'agneaux (8), n'observent pas de modification de la coloration après une supplémentation d'acétate d' $\alpha$-tocophérol incorporé dans l'aliment, à raison de $20 \mathrm{mg} / \mathrm{kg}$ d'aliment. Par ailleurs, Hidiroglou et al (1990) montre l'efficacité d'un apport massif $(160 \mathrm{mg} / \mathrm{kg}$ de poids vif en injection in- trapéritonéale) sur la recharge en vitamine $E$ de divers organes et tissus du mouton. Or, le tissu gras possède aussi la faculté d'accumuler l'a-tocophérol (Bjørneboe et al, 1990). Dans ces conditions, et compte tenu des délais de mise à disponibilité de la vitamine dans les tissus (Hidiroglou et Karpinski, 1988), l'effet d'une surcharge en vitamine $\mathrm{E}$ administrée à des agneaux peu avant l'abattage méritait d'être observé sur le développement des couleurs du tissu gras externe des carcasses.

\section{MATÉRIEL ET MÉTHODES}

\section{Animaux}

Deux cent quarante agneaux mâles Lacaunelait, sevrés précocement (4 à $5 \mathrm{sem}$ ) ont été engraissés intensivement en bergerie (GMQ $=312$ $\mathrm{g} / \mathrm{j} ; \mathrm{CV}=11,2 \%$ ) avec un aliment concentré (1 UFV/kg MS; $112 \mathrm{~g}$ de PDIN/kg MS, $104 \mathrm{~g}$ de $\mathrm{PDIE} / \mathrm{kg}$ de MS). L'abattage des agneaux a été réalisé à degré d'engraissement optimal, apprécié par maniement au cours d'un tri. Quatre jours avant l'abattage, un agneau sur 2 a reçu une dose orale de $4 \mathrm{~g}$ d'acétate de Dlatocophérol (Rhône-Poulenc). Cette quantité a été retenue sur la base des délais de diffusion dans les différents compartiments et de la biodisponibilité de cette forme de tocophérol (Hidiroglou et Karpinski, 1988).

\section{Paramètres observés}

\section{Paramètres zootechniques}

À titre de référence, la vitesse de croissance sur l'ensemble de l'engraissement (GMQ en $\mathrm{g} / \mathrm{j}$ ) et le poids carcasse (Pcar en $\mathrm{kg}$ ) sont rapportés. Plusieurs paramètres d'engraissement externe des carcasses ont été relevés après $18 \mathrm{~h}$ de ressuage en chambre frigorifique $\left(4^{\circ} \mathrm{C}\right)$, en raison de leur liaison avec la coloration du tissu gras : l'importance de l'engraissement externe (NGext) selon la classification Europa, l'épaisseur du gras dorsal (EGdo en $\mathrm{mm}$ ) appréciée à 
partir d'une fente pratiquée au scalpel latéralement aux première et deuxième vertèbres lombaires, la notation de la tenue (NTen) du tissu gras sous-cutané (fermeté et texture) jugée d'après une échelle en 4 points définie par Bouillier-Oudot et al (1992) (NTen 1 = gras mou et huileux; NTen 4 = gras très ferme).

La notation de la coloration (NCoul) a été réalisée selon une échelle de 7 degrés, par référence au nuancier Munsell (Bozzolo et al, 1990).

Un seul et même examinateur expérimenté a procédé aux jugements sur carcasse.

\section{Paramètres chimiques}

La teneur plasmatique en vitamine $E$ a été contrôlée sur 30 agneaux appartenant à chacun des lots (15 traités, 15 témoins). Le prélèvement sanguin a été réalisé par ponction à la veine jugulaire à l'aide de tubes venoject imprégnés d'héparinate de lithium, la veille de l'abattage, soit 3 j après l'administration orale de vitamine E. Les plasmas furent séparés dans l'heure suivant la prise de sang.

L'extraction du tocophérol plasmatique a été effectuée selon le protocole indiqué par Ingold et al (1987). Le dosage a été réalisé par spectrofluorimétrie, selon la technique décrite par Taylor et al (1976).

\section{Traitement des résultats}

Les paramètes quantitatifs sont comparés à partir de leur moyenne à l'aide du test de Student. Les paramètres à caractère ordinal (NTen, NGext) ou qualitatif (Ncoul) sont analysés selon le profil de répartition des fréquences de classe et sont comparés par le test du $\chi^{2}$. La comparajson des fréquences 2 à 2 est réalisée à l'aide du $\chi^{2}$ avec correction de continuité de Yates.

\section{RÉSULTATS ET DISCUSSION}

\section{Caractéristiques de l'échantillon}

Le poids de carcasse, la note de gras externe et l'épaisseur de gras dorsal ne sont pas ou sont très peu différents entre le lot supplémenté en vitamine $E$ et le lot témoin (tableaux I et II). Les poids des carcasses, assez élevés $(19,5 \mathrm{~kg}$ en moyenne) sont identiques et homogènes dans les 2 lots (CV $=7,6 \%$ vs $\mathrm{CV}=6,6 \%)$. Les indicateurs de l'engraissement périphérique (NGext, EGdo) montrent que sur l'en-

Tableau I. Caractéristiques de carcasse et teneur en vitamine $E$ plasmatique selon le traitement reçu par les agneaux (témoin, vitamine E).

\begin{tabular}{lccl}
\hline & Lot témoin (sd) & Lot Vit E (sd) & $S^{*}$ \\
\hline $\begin{array}{l}\text { Paramètres } \\
\text { Effectif }\end{array}$ & 120 & 120 & \\
$\begin{array}{l}\text { Poids carcasse (Pcar, kg) } \\
\text { Importance du gras exteme }\end{array}$ & $19,5(1,3)$ & $19,5(1,5)$ & $\mathrm{NS}$ \\
(NGext 1, Europa) & $2,5(0,6)$ & $2,3(0,5)$ & $P<0,05$ \\
Épaisseur de gras dorsal & $2,2(1,2)$ & $2,5(1,5)$ & $\mathrm{NS}(P<0,15)$ \\
(EGdo, mm) & $2,0(0,7)$ & $2,0(0,7)$ & $\mathrm{NS}$ \\
Note de fermeté (Nten $\left.{ }^{2}\right)$ & & & \\
Teneur plasmatique & 15 & 15 & $P<0,001$ \\
Effectif & $0,70(0,18)$ & $1,93(0,83)$ & \\
Vitamine E $(\mu \mathrm{g} / \mathrm{ml})$ & & & \\
\end{tabular}

" Seuil de signification (test de Student); NS : non significatif; ' NGext : note d'état d'engraissement (grille Europa): 1: maigre; 2 ciré; couvert; 4: gras; 5 : très gras; ${ }^{2}$ Nten: 1: gras mous et hulleux; 4: gras très ferme. 
Tableau II. Qualité du tissu gras sous-cutané des agneaux supplémentés ou non en vitamine E (fréquences relatives).

\begin{tabular}{|c|c|c|c|c|c|c|c|c|}
\hline \multicolumn{9}{|c|}{ Développement du tissu gras externe : NGext (Europa) ${ }^{1}$} \\
\hline & 1 & 1,5 & 2 & 2,5 & 3 & 3,5 & 4 & 4,5 \\
\hline Lot témoin $(n=120)$ & 0,8 & 6,7 & 28,3 & 31,7 & 25,8 & 5,8 & 0 & 0,8 \\
\hline Lot vitamine $\mathrm{E}(n=120)$ & 0 & 1,0 & 34,2 & 35,8 & 17,5 & 1,7 & 0,8 & 0 \\
\hline Ensemble & 0,4 & 8,4 & 31,2 & 33,7 & 21,7 & 3,7 & 0,4 & 0,4 \\
\hline
\end{tabular}

Fermeté du tissu gras externe : $\mathrm{N}$ ten ${ }^{2}$

$\begin{array}{lcccc} & 1 & 2 & 3 & 4 \\ \text { Lot témoin }(n=120) & 22,5 & 53,3 & 24,2 & 0 \\ \text { Lot vitamine } \mathrm{E}(n=120) & 25,0 & 51,7 & 23,4 & 0 \\ \text { Ensemble } & 23,7 & 52,5 & 23,7 & 0\end{array}$

$\chi^{2}(1,2,3)=0,21 ;$ non significatif

Coloration du tissu gras externe : $\mathrm{N} \mathrm{coul}^{3}$

$\begin{array}{llllllll} & 1 & 2 & 3 & 4 & 5 & 6 & 7 \\ \text { Référence Munsell } & 5 Y R 9 / 4 & 7.5 Y R 9 / 4 & 5 Y R 8 / 6 & 7.5 Y R 8 / 6 & 5 Y R 8 / 8 & 10 Y R 9 / 4 & 10 Y R 8 / 6 \\ \text { Lot témoin }(n=120) & 0 & 19,2 & 10,8 & 30,8 & 4,2 & 21,7 & 13,3 \\ \text { Lot vitamine } \mathrm{E}(n=120) & 1,7 & \mathbf{3 8 , 3} & \mathbf{3 , 3} & 22,5 & 0,8 & 22,5 & 10,8 \\ \text { Ensemble } & 0,8 & \mathbf{2 8 , 7} & 7,1 & 26,7 & 2,5 & 22,1 & 12,1\end{array}$

$\chi^{2}(1+2,3,4,5,6,7)=19,44 ; P<0,005$

${ }^{1} \mathrm{~N}$ Gext: note d'état d'engraissement (grille Europa) (1: maigre; 2 : ciré; 3 : couvert; $4:$ gras; $5:$ très gras); ${ }^{2}$ Nten : note de fermeté ( 1 : gras mou et huileux; 4 : gras très ferme); ${ }^{3}$ Ncoul : note de coloration (1: blanc; 2 : blanc crème; 3 : brun-rouge clair; 4 : bistre clair; 5 : brun-rouge; 6 : jaune clair; 7 : bistre avec reflet verdâtre).

semble des agneaux, l'option d'abattage à degré d'engraissement optimal a été respectée (NGext $=2,4 ; 86,7 \%$ des agneaux étant classés entre les notes 2 et 3 ). Les individus du groupe témoin sont cependant en moyenne légèrement plus couverts que ceux du lot traité (NGext $=2,5$ vs 2,3; $P<$ 0,05).

La fermeté du tissu adipeux externe est semblable dans les 2 lots (Nten $=2$; $\mathrm{CV}=$ $35 \%)$. En conséquence, cette variable ne constitue pas un facteur potentiel permettant d'expliquer une différence de fréquence dans la répartition des couleurs. L'observation des profils de répartition des notes de fermeté fait ressortir l'absence de gras très fermes (Nten 4) dans cet échantillon, une majorité de carcasses médiocres (Nten $2=52 \%$ ) et la proportion non négligeable de carcasses au gras mou et huileux (Nten $1=24 \%$ ).

\section{Teneur en vitamine E plasmatique}

La voie d'administration du supplément vitaminique, sa forme chimique (DL $\alpha$ tocophérol), la dose employée (4 g par animal, soit en moyenne $0,1 \mathrm{~g} / \mathrm{kg}$ de poids vif), $4 \mathrm{j}$ avant l'abattage, ont permis d'accroître significativement la vitaminémie : le lot supplémenté présente une teneur plasmatique 2,7 fois plus élevée $(1,93 \mu \mathrm{g} / \mathrm{ml}$ vs $0,70 \mu \mathrm{g} / \mathrm{ml} ; P<0,001$; tableau I). Ces concentrations sont voisines de celles présentées par Hidiroglou et Karpinski (1988). Cependant, la forte variabilité enregistrée parmi les agneaux traités $(\mathrm{CV}=43 \%)$, due 
en particulier à 2 individus présentant de faibles taux de vitamine $E$, traduit un manque d'homogénéité dans la réponse. Celuici est probablement lié au mode d'administration orale (qualité du drogage, interférence avec le bol alimentaire et sa capacité oxydante, niveau d'abreuvement). Hidiroglou et al (1990) signalent l'excellente efficacité de la voie intrapéritonéale. Ce mode de traitement permettrait sans doute de mieux maitriser les doses réellement apportées et de réduire cette source de variabilité.

\section{Coloration du tissu gras}

If ressort du tableau II que l'influence du traitement par administration de vitamine $E$ est très significative $(P<0,004)$ sur la notation de la couleur du tissu gras externe, variable impliquée dans cet essai. Les proportions de carcasses de couleur blanccrème sont accrues (NCoul 2 [7.5YR 9/4] = $38,3 \%$ vs $19,2 \%$ pour le lot témoin; $P<$ 0,001 ) au détriment des colorations à tendance bistre-rougeâtre (Ncoul 3 [5YR 8/6] + Ncoul 4 [75YR 8/6] + Ncoul 5 [5YR 8/8] = $26,6 \%$ vs $45,8 \% ; P<0,002$ ). Au contraire, les graisses à nuance jaune (Ncoul 6 [10YR 9/4] et Ncoul 7 [10YR 8/6]) ne sont pas affectées. Sur l'ensemble de la gamme de coloration, nous notons la prépondérance des classes de couleur 2, 4 et 6, c'est-à-dire : blanc-crème, bistre-clair et jaune-clair, soit respectivement pour l'échantillon total : $28,7 \%, 26,7 \%$ et $22,1 \%$. La faible proportion de carcasses blanches (Ncoul 1) est à mettre en relation avec l'absence de tenue très ferme (Nten 4). Par rapport à la répartition des colorations habituellement constatée pour cette catégorie d'agneaux (Bozzolo et al, 1991), le profil observé sur le lot témoin est cohérent : nous retrouvons, en particulier $19 \%$ de couleur blanc-crème, $31 \%$ de couleur bistre, $22 \%$ de couleur jaune claire. Cette influence significative de la vitamine $E$, contrairement aux résultats de Murphy et L'Estrange (1977), peut être expliquée par la forte dose employée et le moment du traitement réalisé peu de temps avant l'abattage, permettant une meilleure efficacité antioxydante de l'acétate de tocophérol.

La modification des fréquences de coloration repose plus particulièrement sur les nuances Ncoul 2 déjà mentionnée, et Ncoul $4(22,5 \%$ vs $30,8 \%$ pour le lot témoin). Un facteur explicatif possible pourrait consister en la limitation des phénomènes oxidatifs pour une part des individus habituellement classés en 4 . Le pourcentage résiduel de carcasses $(22,5 \%)$ demeurant classé Ncoul 4 après traitement peut traduire, partiellement, un manque d'efficacité de l'administration orale utilisée (qualité du drogage). Il peut aussi relever d'autres mécanismes non élucidés comme l'accumulation de pigments héminiques (Prache et al, 1990) en liaison avec la composition en acides gras insaturés des phospholipides, susceptibles de fragiliser les membranes.

\section{CONCLUSION}

Le passage des couleurs de type blanccrème au type bistre au cours du ressuage pourrait relever d'un phénomène partiel d'oxydation des graisses insaturées. Cette évolution serait limitée par la présence de vitamine E. La meilleure stabilité des pigments héminiques présents pourrait être aussi à l'origine de cette réduction de l'oxydation (Faustman et al, 1989) en raison de leur rôle catalytique dans ce processus (Buckley et al, 1989). En revanche, nous n'observons pas de modification pour les colorations à tendance jaune (Ncoul 6 et 7). Ces dernières couleurs peuvent être engendrées par d'autres causes que l'oxy- 
dation comme l'hyperbilirubinémie ou l'accumulation de pigments caroténoïdes (Rozier et al, 1979; Gouband, 1982). Dans ce cas, l'aspect génétique peut être essentiel ainsi que le laissent supposer les observations de Kirton et al (1975) et Baker et al (1985), dès lors qu'il ne s'agit pas d'une atteinte ictérique pathologique (Popoff et al, 1980).

L'administration de vitamine $E$, malgré son effet positif sur la présentation des carcasses, ne constitue cependant qu'un palliatif, et il semble essentiel de parvenir à limiter l'importance des acides gras incriminés dans ce phénomène oxydatif.

\section{REMERCIEMENTS}

Ce travail a été permis grâce à l'aide apportée par le Conseil régional de Midi-Pyrénées (contrat $n^{\circ}: 9000743$ ). La préparation de DL $\alpha$ tocophérol a été gracieusement offerte par Rhône-Poulenc Animal Nutrition.

\section{RÉFÉRENCES}

Baker RL, Steine T, Vabeno AW, Breines D (1985) The inheritance and incidence of yellow fat in nowwegian sheep. Acta Agric Scand 35, 389-397

Bartov I, Basker D, Angel S (1983) Effect of dietary vitamin $E$ on the stability and sensory quality of Turkey meat. Poult Sci 62, 12241230

Bjørneboe A, Djømeboe GEA, Drevon CA (1990) Absorption, transport and distribution of vitamin E. J Nutr 120, 233-242

Bouillier-Oudot M, Bozzolo G, Phrem G, Grasset D, Manse $H$ (1992) Optimisation du jugement de la fermeté du gras de couverture des carcasses d'agneaux de bergerie. Ann Zootech 41 (2), 187-203

Bozzolo G, Bouillier-Oudot M, De Boisseson E, Ghassan M, Grasset D (1990) Influence des performances zootechniques sur les caractéristiques des tissus adipeux d'agneaux de bergerie, sevrés précocement et alimentés avec un régime à forte concentration énergétique. Ann Zootech 39, 77-94

Bozzolo G, Bouillier-Oudot M, Aoun M, Grasset $D$, Manse $H$ (1991) Incidence des acides gras alimentaires à chaîne moyenne, incorporés dans le régime de transition en postsevrage, sur les caractéristiques de croissance et de carcasse d'agneaux sevrés précocement et engraissés intensivement en bergerie en été. Ann Zootech 40, 85-104

Buckley DJ, Gray JI, Asghar A, Price JF, Crackel RL, Booren AM, Pearson AM, Miller ER (1989) Effects of dietary antioxidants and oxidized oil on membranal lipid stability and Pork product quality. J Food Sci 54, 11931197

Faustman C, Cassens RG, Schaefer DM, Buege DA, Williams SN, Scheller KK (1989) Improvement of pigment and lipid stability in Holstein steer beef by dietary supplementation with vitamin E. J Food Sci 54, 858-862

Gouband F (1982) Les graisses molles des agneaux. Thèse vét ENV Alfort, Université Paris-Créteil, $125 \mathrm{p}$

Hidiroglou M, Karpinski K (1988) Pharmacokinetic disposition in sheep of various vitamin $E$ preparations given orally or intravenously. $\mathrm{Br}$ J Nutr 59, 509-518

Hidiroglou N, Butler G, Mc Dowell LR (1990) Plasma and tissue vitamin $E$ concentrations in sheep after administration of a single intraperitoneal dose of DL $\alpha$ tocopherol. J Anim Sci $68,782-787$

Ingold KU, Burton GW, Foster DO, Hughes L, Lindsay DA, Webb A (1987) Biokinetics of and discrimination between dietary RRR- and SRR- $\alpha$ tocopherols in the male rat. Lipids 22, 163-172

Kirton AH, Crane B, Patterson DJ, Clare NT (1975) Yellow fat in lambs caused by carotenoid pigmentation. NZ J Agric Res 18, 267272

Leboulanger J (1977) Les vitamines. Brochure Roche, 61-68

Lehninger AL (1979) Biochimie - Vitamines et coenzymes. In : Bases moléculaires de la structure et des fonctions cellulaires. Flammarion Médecine, France, 331-356

Murphy J, L'Estrange JL (1977) The performance and carcass fat characteristics of 
lambs fattened on concentrate diets. 1. Effect of maize and barley as the cereal source and of dietary supplementation with roughage, vitamin E, cobalt and vitamin B12. Ir J Agric Res 16, 187-204

Palmer DN, Husbands DR, Winter PJ, Blunt JW, Joly RD (1986) Ceroid lipofuscinosis in sheep. I-phospholipids, fatty acids and fluorescence in liver lipopigment lipids. $J$ Biol Chem 261, 1766-1772

Popoff M, Meneroud M, Petit A, Tassin P, Belinsky $C$, Lalou-Keraly FX, Christodoulakis $F$ (1980) Carcasses d'ovins à coloration jaune. Etudes bactériologiques et chimiques. Bull Soc Vét Prat Fr 64 (2), 155-171
Prache $S$, Aurousseau $B$, Thériez $M$, Renerre M (1990) Les défauts de couleur du tissu adipeux sous-cutané des carcasses d'ovins. INRA Prod Anim 3, 275-285

Rethwill C, Bruin TK, Waibel PE, Addis PB (1981) Influence of dietary fat source and vitamin $E$ on market stability of turkeys. Poultry Sci 60, 2466-2474

Rozier J, Ferrando R, Cartier V, Fourlon C (1979) Nature des graisses jaunes chez le mouton. Bull Acad Vét Fr 52, 545-552

Taylor SL, Lamben MP, Tappel AL (1976) Sensitive fluorimetric method for tissue tocopherol analysis. Lipids 11, 530-538 